\title{
The research of the sun shadow positioning
}

\author{
Lingyu Huang \\ School of North China Electric Power University, Baoding 071000, China
}

\begin{abstract}
In recent decades, with the level of material life improves quickly, more and more people are on fire for outdoor adventure. They usually trek in desert and wasteland. However the case that compass or communication equipment is out of order happens frequently. For someone who lack direction, it is easy for them to be lost. So it is a meaningful work to research a way to find position with the help of the sun shadow. The sun shadow position is a huge project. Our paper aims to solve one link of the whole project. As the time and location change, the length of pole's shadow change, which we make research on to get the exact function model. As is known to all, earth-sun distance is much higher than mean radius of the earth. So we can approximately think the sunlight shining on the earth is parallel light. If we believe pole length is constant. The shadow length only relates with solar elevation angle. So we can use solar declination angle, solar hour angle, geographical longitude and latitude to calculate solar elevation angle. In a word, we use parameters such as date, longitude, latitude and time to refer to above variable, with the help of geographical knowledge. And then we establish the model which describe the change of shadow length. At last we apply the model in practice. The result prove that our model is reasonable.
\end{abstract}

KEYWORD: shadow positioning; solar elevation; shadow length change model

\section{INTRODUCTION}

With the rapid development of technology, we may lose many skills that our ancestors master. For example, we may well lose direction in the desert, if we don't have navigator. While our ancestors can distinguish the orientation with the help of shadow. In addition, with the rise of video, more and more people want to distinguish the data that when and where photo are taken. So, we consider it significant to research how to distinguish orientation with the help of pole's length. Our paper does some work in this aspect.

\section{ASSUMPTION}

1.when the sunlight shining into earth, we take no account of refraction.

2.The difference in terrain don't influence our result.(like high mountain and basin)

\section{ESTABLISHMENT OF MODEL}

Because earth-sun distance is very far, we can approximately think the sunlight shining on the earth is parallel light in the field of optical. As shown, we can know the shadow length if we have known the pole's length and the angle between sunlight and ground plane. We call the angle .In other words, we can get equation which describe the change of shadow length, if we know how change with time.

Following is the formula about shadow length.

$l=\frac{h}{\tan \theta}$

These letters represent specific meaning. $l$ means the length of pole's shadow. $h$ means the height of pole. The meaning of $\theta$ will expound below. 


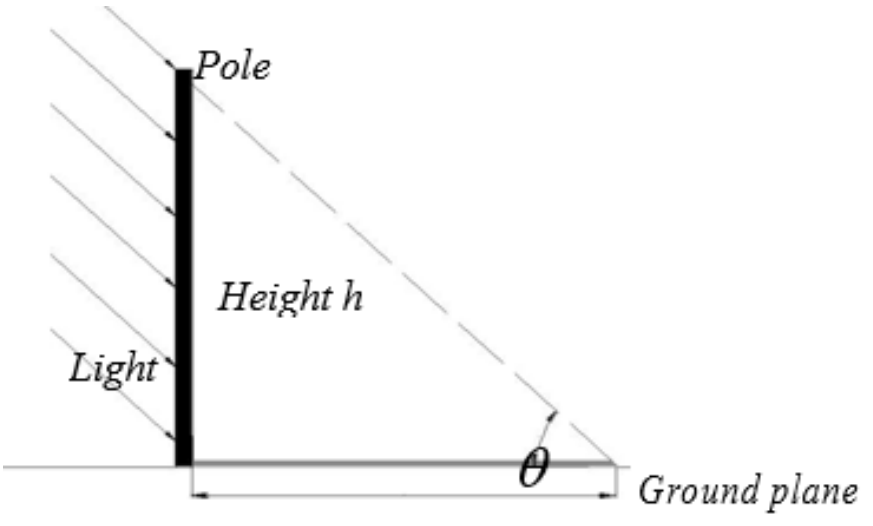

Shadow length $l$

Graph 1. sketch map

\section{HOW TO CALCULATE $\theta$}

By looking at data, we discover the truth that $\theta$ are defined strictly. $\theta$ represent solar elevation angle. Solar elevation angle refer to the angle between solar ray and the earth's surface tangent. At high noon, the solar elevation angle reach maximum during one day. At the same time, the shadow length reach minimum during one day.

Following is the formula about solar elevation:

$\sin \theta=\sin \delta \sin \varphi+\cos \delta \cos \varphi \cos \tau$

Refer to solar declination angle, $\varphi$ represents the latitude of the area where the pole lie. $\tau$ means hour angle of true sun.

\section{SOLAR DECLINATION ANGLE $\delta$}

Solar declination angle means the angle between sun-earth center's line and equatorial plane. In geography, we call orbital plane where the earth revolves round the sun ecliptic plane. The solar declination angle appears because polar axis and ecliptic plane are not vertical. Next, we will give the computational formula.

$$
\delta=23.45 \times \sin \left[(n-80.25) \times\left(1-\frac{n}{9500}\right)\right]
$$

$N$ means the date's sequence number in one year.

\section{HOUR ANGLE OF TRUE SUN $\tau$}

We define the true solar day as time interval that the sun pass upper culmination for successive two times. On the basis of the true solar day, the time we calculate is true solar time. However, people believe the timing system should be balance and stable in daily life. So they assume a theory that the sun moves at constant speed around the equator. The period is one year. In this theory, corresponding time is mean solar day and mean solar time. Obviously, we can calculate true solar time through mean solar time. Moreover, we should consider time difference. Take China for example, China take eastern eight zone time as local standard time. So we should think about time difference when we calculate local time in other areas. If we believe true solar time is local time, we can get formula to calculate true solar time.

$$
S_{\odot}=S_{d}=S+\left\{F-\left(120^{\circ}-\phi\right) \times 4\right\} / 60
$$

$S_{\odot}$ is true solar time, $S_{d}$ means local time. $\mathrm{S}$ and $F$ represent hour and minute in local standard time system, $\phi$ is latitude.

To get more accurate true solar time, considering the fact that solar annual motion is nonuniform, we amend the true solar time in Beijing. And then we get more accurate formula.

$S_{\odot}=S_{d}+E_{t}$

$E_{t}$ is correction.Following .is modifier formulas.

$E_{t}=229.2 \times(0.000075+0.001868 \cos B-0.032077 \sin B$

$-0.014615 \cos 2 B-0.014615 \cos 2 B-0.04089 \sin 2 B)$

$B=\frac{(n-1) \times 360}{365} N$ means the date's sequence number in one year.

From this, we can get the way to calculate solar hour angle $\tau$.

$\tau=\left(S_{\odot}-12\right) \times 15$

In conclusion, according to equation above, we can confirm solar elevation angle. Therefor, if we have known an exact date and site (latitude and longitude),we can calculate corresponding $\theta$.in other words, we can use above-mentioned parameter to simplify shadow length.

$l=f(h, j, f, n, S, F)$

Following is specific equation set.

$\left\{\begin{array}{l}\delta=23.45 \times \sin \left[(n-80.25) \times\left(1-\frac{n}{9500}\right)\right] \\ \tau=\left(S_{\odot}-12\right) \times 15 \\ S_{\odot}=S_{d}+E_{t} \\ S_{d}=S+\left\{F-\left(120^{\circ}-\phi\right) \times 4\right\} / 60 \\ \sin \theta=\sin \delta \sin \varphi+\cos \delta \cos \varphi \cos \tau \\ l=\frac{h}{\tan \theta}\end{array}\right.$

We verify model's rationality with the help of geographic knowledge and commonsense knowledge. The result shows that change rule of shadow length is as same as what we predict. To some extent, we 
can believe our model is reasonable. And then, we will apply our model in a certain situation, to test model's rationality.

\section{MODEL APPLICATION AND THE RESULT}

We choose one case as example. Following is our given information. The date is October 22, 2015.The place is Tian An Men square in Beijing (The northern latitude 39 degrees 54 minutes 26 seconds and the east longitude 116 degrees 23 minutes 29 seconds). The length of pole is 3 meters. The time interval we concern is from 9 am to $3 \mathrm{pm}$. Then we use the model we establish to get a curve that represent the change of pole's shadow length, with the help of matrix laboratory. The curve is shown in graph 2 .

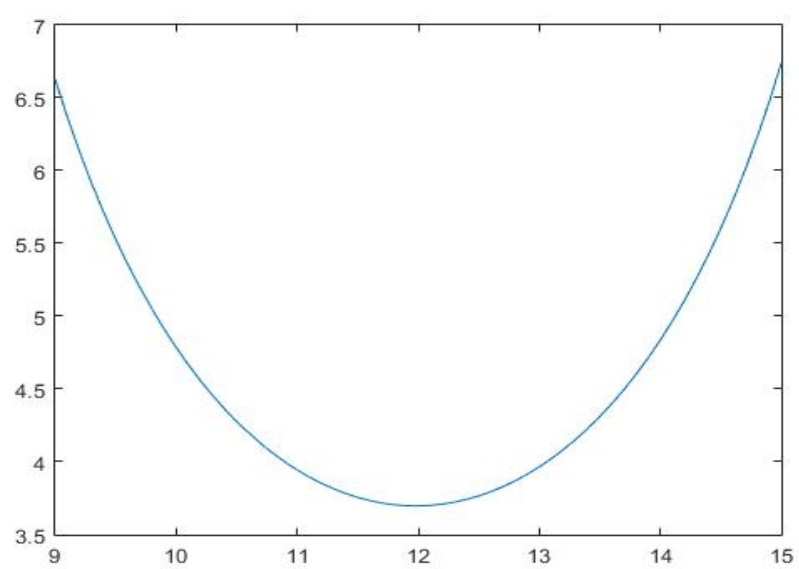

Graph 2.the curve of shadow length

In graph 2, abscissa represents Beijing time and ordinate represents shadow length. In table 1, we give shadow length at the whole point of time. Combining graph 2 and table 1, we could come to know the fact that shadow length is the shortest at noon. As time goes on, the shadow length is getting longer. For our case, the shortest shadow length is between 3.6 meters and 3.7 meters.

Table 1.Shadow length at the whole point of time

\begin{tabular}{cccccccc}
\hline Beijing Time & $9: 00$ & $10: 00$ & $11: 00$ & $12: 00$ & $13: 00$ & $14: 00$ & $15: 00$ \\
\hline Shadow length(m) & 6.6275 & 4.785 & 3.9438 & 3.6976 & 3.9652 & 4.8358 & 6.7391 \\
\hline
\end{tabular}

After consulting information, we know the truth that solar elevation angle is around 37 degree at noon in October, in Beijing. Namely, the pole's shortest shadow length is around 3.98 meters. It shows that our result is similar to the fact. The result prove that our model is reasonable once again.

\section{THE CONCLUSION}

Combining geography and mathematical knowledge, we give an accurate algorithm to calculate shadow length according the information about location and time at last. This work makes sense, because it lays the foundation of next work. In the future, we can continue to explore the method how we judge orientation with the help of change of shadow length. We still have a lot of work to do.

\section{REFERENCES}

[1] Shougui Si, Xi Sun. Mathematical modeling algorithm and application. Beijing: National Defence Industry Press, 2015.2.

[2] Qiyuan Jiang, Jinxing Xie. Mathematical model. Beijing: Higher Education Press, 2011.

[3] Bingzhong wang. Lecture on calculation of solar radiation: the Fifth lecture. Calculation of radiation on the surface ramp [J]. Solar Power, 2000(3):20-21.
[4] Haibo Liu, Jiangfang Wang, Haiqin Yu. Calculation and application of several related angles in solar energy projects [J]. China Construction Dynamics: Sunshine Energy, 2010, (6): $67-70$ 